\title{
Notch Family
}

National Cancer Institute

\section{Source}

National Cancer Institute. Notch Family. NCI Thesaurus. Code C113838.

A family of transmembrane receptors with multiple extracellular EGF repeats and a DSL

(notch) domain. These proteins interact with their cell-bound ligands (from the delta and serrate protein families). This interaction stimulates cell-cell signaling pathways that regulate cell fate decisions during embryogenesis. 